\title{
GENETICALLY CONTROLLED DEGENERATION OF THE NUCLEUS PULPOSUS IN THE MOUSE
}

\author{
R. J. BERry, LONDON, ENGLAND \\ From the Medical Research Council Experimental Genetics Research Unit, \\ University College, London
}

In the past few years ideas on the etiology of intervertebral disc protrusion have undergone a fairly radical change, and "it is now generally conceded that trauma is not a large factor in the production of disc prolapse, and may often be absent" (Annotation, Lancet 1958). As long ago as 1948 Jackson suggested that there are anatomical defects which predispose to disc protrusion and which are "most likely of a developmental nature, possibly increased by other levers in the form of trauma, toxaemia and pregnancy." Histochemical and biophysical studies of the discs (Charnley 1952, Malinský 1957, Hendry 1958, Naylor 1958) seem to point to the conclusion that predisposing weaknesses in the discs may be regarded as similar to normal ageing changes. However, " gross degeneration in older people is rarely associated with prolapse. If desiccation in discs is a major factor in their prolapse it is difficult to understand why the elderly remain relatively free from major disc derangements " (Annotation, Lancet 1958). This fact must remain unexplained unless it can be shown that certain people have an inherent proneness to disc lesions, or, in other words, unless it is found possible to divide the population into those whose discs show such changes during the first twenty or thirty years of life that they are predisposed to prolapse, and those whose discs undergo only "normal" changes during life.

However, just such changes have been shown in at least two experimental animals. Hansen $(1951,1952)$ showed that markedly different rates of disc prolapse exist in different breeds of dogs, and that prolapse in these animals is certainly due to degenerative changes in the nucleus pulposus. The purpose of this communication is to describe a similar genetically determined effect in the house mouse, resulting from a progressive reduction of the notochord in a cephalo-caudad direction (Berry 1960).

\section{THE PINTAIL MUTATION IN THE MOUSE}

The work described in this paper refers to a mutation which was found in a mouse of the $\mathrm{pBr}$ strain which had been treated with the powerful carcinogen-and alleged mouse mutagen-methylcholanthrene (Hollander and Strong 1951). The tail of this mouse was about "two-thirds normal length, and the distal end showed a peculiar thin and somewhat distorted tip." The name "Pintail" (symbol $P t$ ) was given to this trait. The gene normally behaves as a partial dominant, and mice heterozygous $(P t /+)$ and homozygous $(P t \mid P t)$ for the gene are fertile. Homozygous Pintail mice have hardly any tail and are often debilitated; they frequently have intestinal abnormalities.

Pintail mice usually have one or more kinks in the distal part of the tail (Fig. 2), but several Pintail heterozygotes have occurred with no tail kinking. The probable explanation is that the tail kinks are the incidental result of abnormal intervertebral discs which are insufficient to maintain the vertebrae in their correct relationship to one another. This conclusion is strengthened by the observation that tail kinks only appear at about the age of two weeks, at which time the mice leave the nest and run around actively.

\section{THE EFFECT OF THE PINTAIL GENE ON THE INTERVERTEBRAL DISCS}

The Pintail gene has an effect on the intervertebral discs which is not obvious from an examination of the living animals, but which is only revealed by histological techniques. 
One of these techniques must be described. Grüneberg (1958) in a personal communication observed that the mucoid nucleus pulposus is stained very clearly in methylene blue clearance preparations (Noback 1916, Grüneberg 1953). This observation was utilised and the axial skeleton of a number of normal and abnormal animals aged three weeks and older was stained

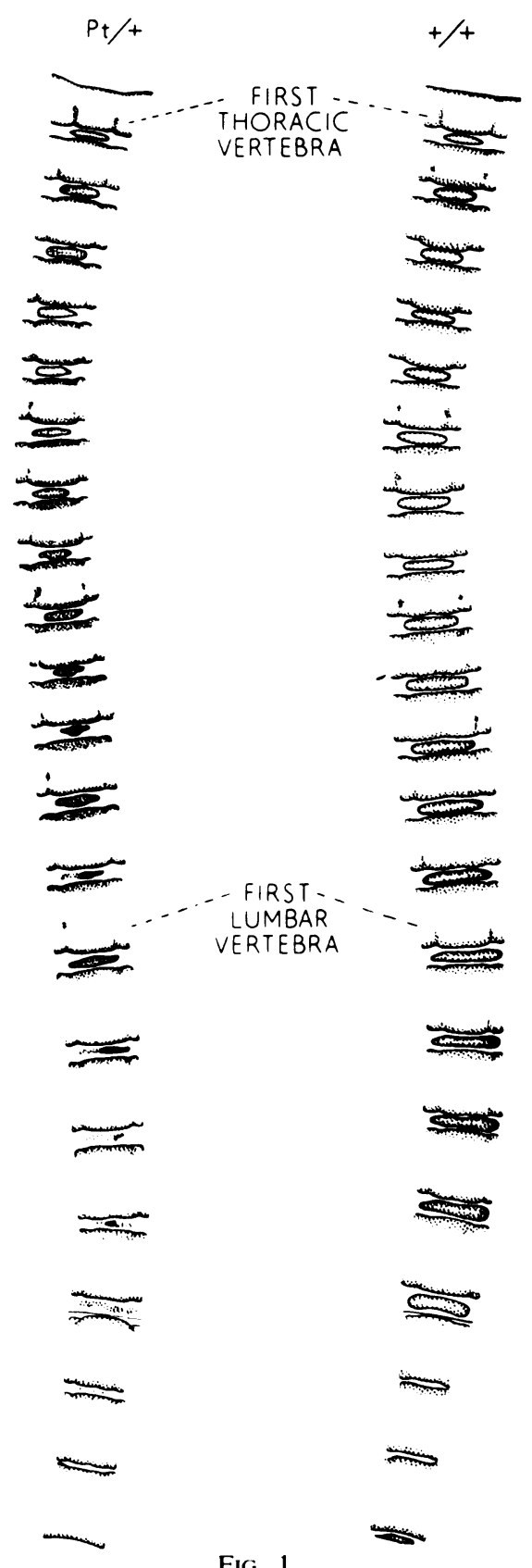

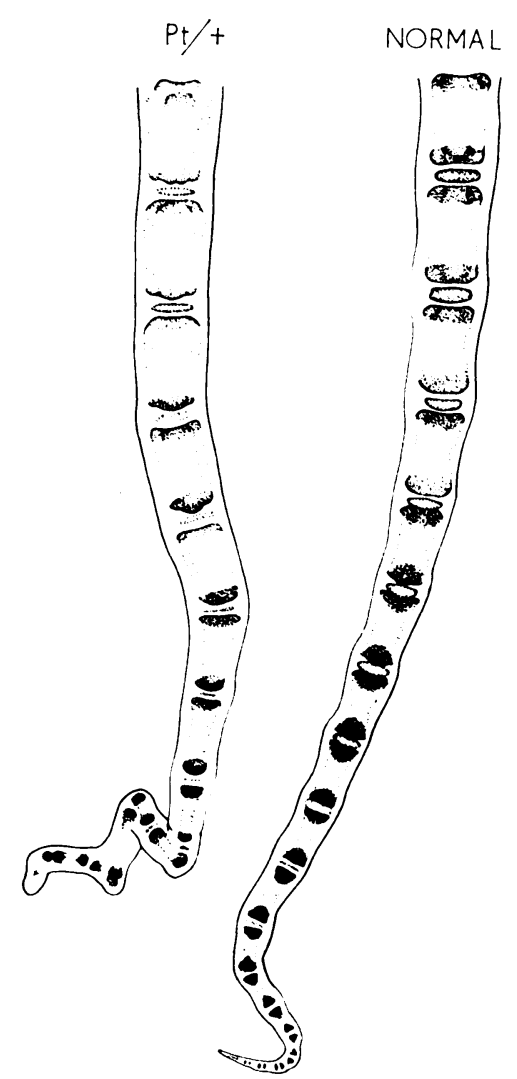

FIG. 2

Figure 1-Freehand drawings of methylene blue stained clearance preparations of the vertebral column of $P t / 4$ (left) and $+-1+($ right $)$ litter mates at twenty-five days old to show the variation in size and shape of the nuclei pulposi.

Figure 2-Freehand drawings of the distal two-thirds of the tails of mice from the same litter as those illustrated in Figure 1. (Reproduced from Genetical Research, 1, 1960.)

to show the nucleus pulposus. The animals were killed and the vertebral column removed, straightened and tied to a glass plate, and fixed in formol saline. The specimens were stained with methylene blue, dehydrated with alcohol and cleared in methyl salicylate. The best preparations are those of animals aged about three weeks. Soon afterwards the affinity of 
the nuclei pulposi in the trunk region for the stain diminishes. This decrease in staining property is rather quicker in Pintails than in normals of the same age, but by the age of eight weeks or so the discs in the cervical region of both genotypes stain hardly at all.

There are differences in both the size and staining intensity of the nuclei pulposi in normals and Pintails (Figs. 1 and 2). The nuclei pulposi in the cervical region are more or less normal. More caudad the size of the nucleus pulposus progressively decreases. The nuclei pulposi in the normal mouse are more or less evenly stained the whole way down the vertebral column; in the Pintail the staining becomes more intense as the discs are reduced in size. This increase in staining intensity is accompanied by a decrease in the definition of the staining boundary of each nucleus pulposus, particularly in the tail, and the shape also changes in a characteristic fashion from head to tail. Between the cervical vertebrae in both normal and Pintail mice the nuclei pulposi are roughly cigar shaped as seen in a thoraco-ventral projection, in the thoracic region those of Pintails are more spherical than those of normals. and in the lumbar region they are flatter and thinner-but the outlines of some of those more caudad are not completely regular. The nuclei pulposi in normal mice reach their greatest size in the lower lumbar region. There are no nuclei pulposi between the first three sacral vertebrae.

\section{MEASUREMENT OF THE NUCLEI PULPOSI}

In an attempt to make the above observations quantitative, the two main diameters of the nucleus pulposus in clearance preparations were measured under a microscope fitted with

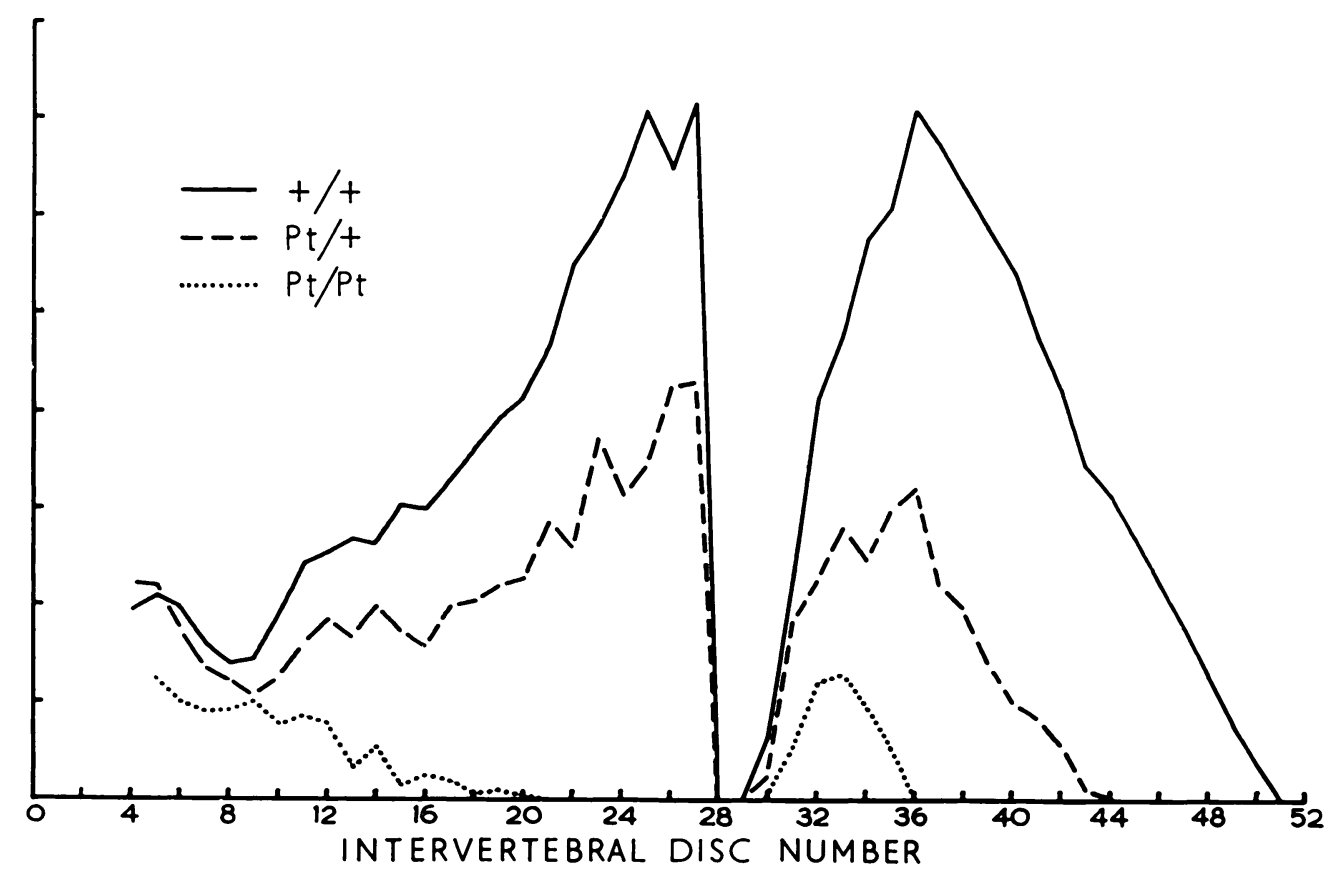

FIG. 3

Variation in size of the nucleus pulposus along the vertebral column. The vertical ordinate is the product of the two main diameters in arbitrary units. Mean values of a litter of two $+\ldots$ (continuous line), three Pt (broken line) and one Pt'Pt (dotted line) animals aged twenty-one days.

a Leitz micrometer screw objective. It was possible to measure these dimensions for almost the whole of the length of the vertebral column, although the diffuse boundary of the staining in the last few tail discs made accurate measurement difficult. Measurements were made upon a series of litters containing normal, $P t /+$, and $P t / P t$ animals. All three genotypes 
had a fairly constant pattern of size variation along the column, although there was quite a lot of both inter-and intra-litter variation. The product of the two diameters was found best to characterise each nucleus pulposus. The sizes of the nuclei pulposi in all the genotypes

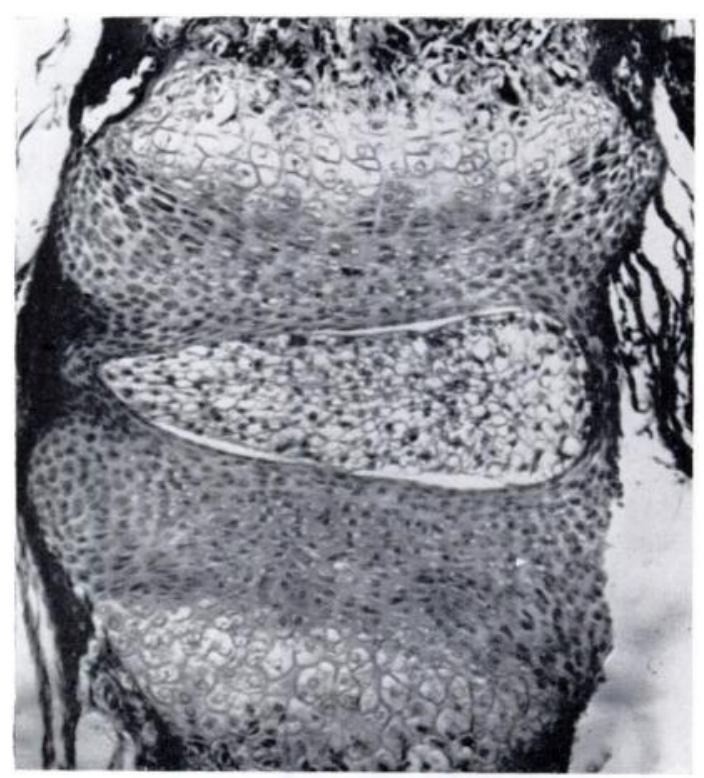

FIG. 4

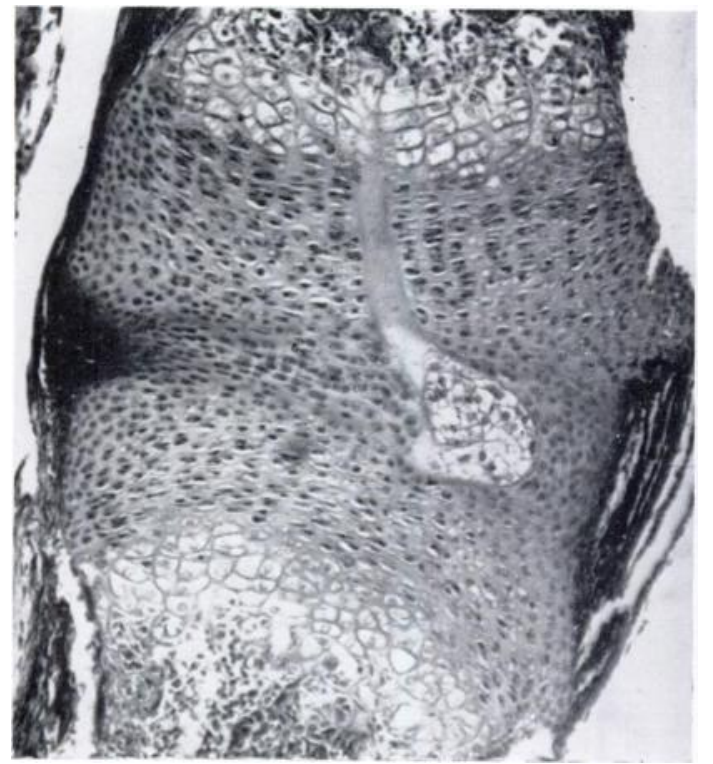

FIG. 5

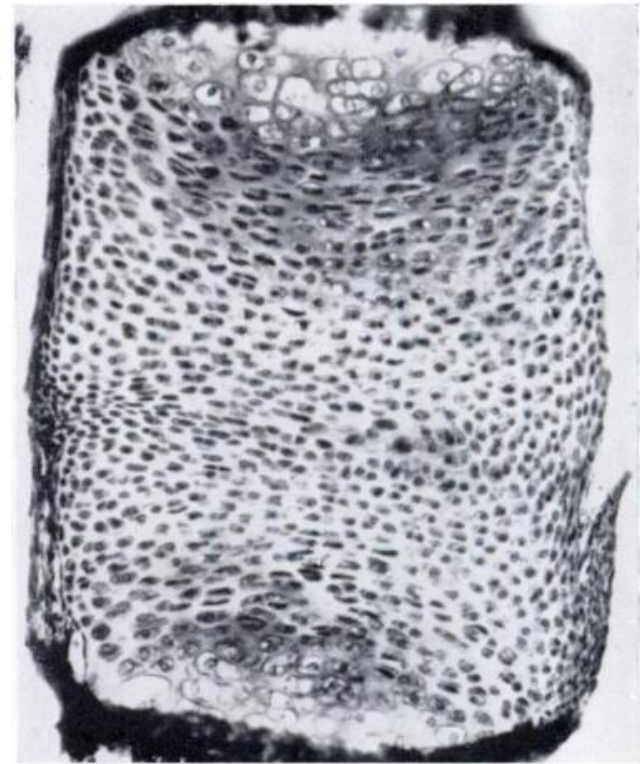

FIG. 6

Figs. 4 To 6

Photomicrographs of sagittal $10 \mu$ sections through lumbar intervertebral discs stained with alcian blue and chlorantine fast red of newborn - (Fig. 4), Pt + (Fig. 5) and Pt Pt (Fig. 6) litter mates. ( . 115.)

were of the same order in the cervical region; from about the tenth disc caudad, the abnormals showed a marked reduction in size; in the Pintail homozygote there were virtually no nuclei pulposi in the posterior thoracic and lumbar regions. The progressive nature of the reduction in size along the vertebral column can be clearly seen in Figure 3. 


\section{HISTOLOGICAL CHANGES}

The consequences of this reduction in size of the nuclei pulposi were investigated histologically. Because of its medical interest, the lumbo-sacral section of the spine was studied in preference to others. The material was fixed in Bouin`s fluid, decalcified with 2 per cent nitric acid. embedded by Peterfi's technique and serially sectioned at $10 \mu$ in the sagittal plane. The sections were stained with Ehrlich's haematoxylin and eosin, or alcian blue and chlorantine fast red (Lison 1954).

At birth (Figs. 4 to 6 ) the nucleus pulposus was little differentiated: in both normal and $P{ }^{\prime}$. mice it consisted of simple aggregations of notochordal cells, which were well stained and whose cell wall reticulum was unbroken. The first signs of acid mucopolysaccharides (stained with alcian blue) penetrating into the cellular structure were apparent, and this process seemed to have advanced farther in the abnormal than in the normal. In conformity

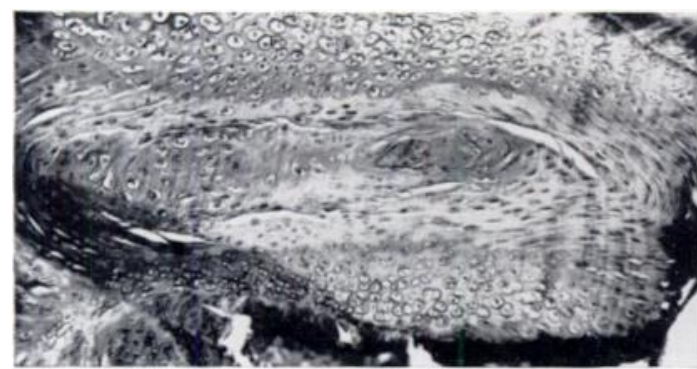

Fig. 7

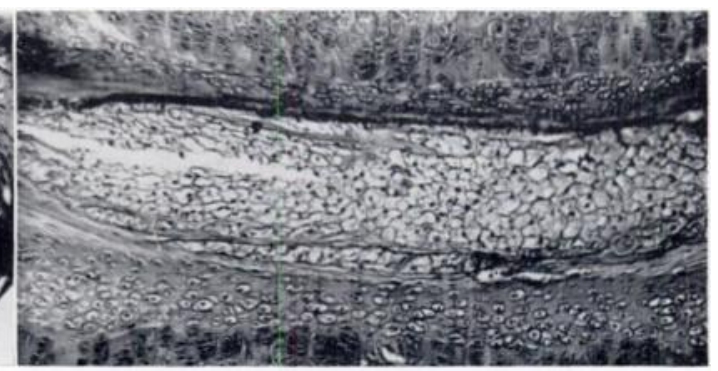

FiG. 8

Similar sections to those shown in Figures 4 to 6 to show the more advanced changes in the nucleus pulposus of the abnormal in fifty-day-old $P t \quad$ (Fig. 7) and : : (Fig. 8) litter mates. $(\therefore 80$.

with the findings in clearance preparations there was no sign of any nucleus pulposus in the $P t / P t$ animal, though traces of the notochordal sheath were found in some sections. The actual disc region consisted mainly of cartilaginous tissue with much acid mucopolysaccharide content, but the tissue was freely interspersed with collagen fibres (stained red with chlorantine fast red).

The penetration of acid mucopolysaccharides into the nucleus pulposus proceeded rapidly during early post-natal life. Already at five days it was clearly surrounded by a zone of mucoid material containing no nuclei, and the penetration of this substance between the cells of the primitive nucleus pulposus had been sufficient to isolate islands of cells. The cell nuclei in $P I_{l}$ - are enlarged and stained less well than at birth. By ten days the whole of the small cellular nucleus pulposus of $P t /+$ was divided into blocks surrounded by homogeneous mucoid substance, and there was more collagen in the surrounding regions.

No trace of the original notochordal cell structure was left in a thirty-nine-day-old $P_{t} \mid-$. The central disc area stained badly with haematoxylin and eosin, but seemed to contain many fibrocytes. A definite central nucleus pulposus existed at fifty days (Fig. 7). This area was almost entirely mucoid with many large nuclei. It was not clear whether these were the remains of notochordal cells, or were fibrocytes and cartilage cells that had immigrated from outside. There was no sign of the original cell wall reticulum. In the normal at this age Fig. 8) the original cell walls were still present and the nuclei clearly visible, and the most obvious change from the early post-natal structure was in the continuing ingression of the cellular area, with some cells seeming to have been confined in vacuoles in the mucoid substance, and the nuclei of these cells showed some signs of disintegration. The annulus fibrosus of both normal and Pintail discs seemed to differentiate normally.

Between fifty and a hundred days the mucoid structure of the $P t /$ disc was largely infiltrated with collagen. Ossification of parts of the annulus fibrosus on the inside of the vertebral end-plates sometimes occurred.

vol. 43 B, NO. 2, MAY 1961 
There were no further major changes. At 250 days the $P t \mid+$ disc was more fibrous than formerly, while the cell wall reticulum of the normal was still clearly visible, although the nuclei were now only just discernible. In fact the lumbar discs of $P t /+$ animals changed more in the first seven weeks after birth than did those of normals in the first seven months.

\section{DISCUSSION}

The effect of the Pintail gene on development has been shown (Berry 1960) to be a reduction in the mitotic rate in the notochord beginning on the tenth day of gestation (birth takes place on the nineteenth or twentieth day). The only essential difference between newborn normal and Pintail mice is that there are more notochordal cells in the former. Evidently the greatly accelerated age changes in the discs of Pintail mice are a direct consequence of their reduced size.

Peacock (1952) and Malinský (1959) have described the changes that take place in the discs of man with increasing age. The main respect in which they differ from those in the mouse is in the more rapid disappearance of notochordal cells. The work of Hansen $(1951,1952)$ on the different dispositions of certain breeds of dog to disc degeneration and prolapse has already been mentioned. The age changes in the discs of breeds subject to disc degeneration closely resemble those in Pintail mice. This condition is a breed characteristic in dogs and hence presumably genetic though it is uncertain whether it is produced by a single gene as is Pintail in the mouse.

Many authors have studied the changes in protruded discs in man. Eckert and Decker (1947) found differences between discs removed at operation and those taken from cadavers of similar ages, the most striking being stroma degeneration of the nucleus pulposus in the operatively removed discs. Hendry (1958) showed that protruded discs in man have a lowered capacity to take up water; he ascribed this to the " loss of or deterioration in the muco-protein gel of the nucleus - that is, the same changes as have been shown by Sylvén (1951) and others to occur normally only with advancing years." Mitchell, Hendry and Billewicz (1961) have shown that the polysaccharide content of a series of prolapsed discs removed at operation was "strikingly uniform" and lower than that of a comparable series of normal discs, in which the polysaccharide content varies with age, declining from the fourth decade onwards. Mice which carry the $P t$ gene show just such a loss of muco-protein in the nucleus pulposus, that is, they exhibit a genetically determined weakness of the intervertebral discs.

The suggestion put forward in this paper is that disc protrusion often, if not always, results from a congenital weakness of the discs themselves. The direct proof of the genetic basis of such a weakness in man would be very difficult: a predisposition to disc protrusion in the third or fourth decade of life is not a subject which lends itself easily to family studies, particularly since there are sex and occupational differences in the incidence of the condition. There may be a gene similar to Pintail widespread in human populations. It would presumably manifest itself in no other way than giving its possessors a "weak back." However, a genetical hypothesis answers the difficulty expressed by the Lancet annotation (1958), that " it is difficult to understand why the elderly remain relatively free from major disc derangements." If there is a human gene which causes accelerated ageing changes, carriers of it would be particularly liable to disc lesions comparatively early in life. People without such a gene for premature ageing would reach a similar degree of disc degeneration only at an age when their relative immobility would tend to protect them from injury.

\section{SUMMARY}

A description is given of a mutation in the mouse which reduces the size of the nucleus pulposus in the intervertebral disc of the adults. The discs of these mice show greatly accelerated age changes. The consequences of a similar mutation in man are discussed.

I am indebted to Professor Hans Grüneberg, F.R.S., for his constant encouragement and criticism, to Miss June Denny for the photography, and to Mr A. J. Lee for the drawings. The work was supported by a grant from the Medical Research Council which is gratefully acknowledged. 


\section{REFERENCES}

Berry, R. J. (1960): Genetical Studies on the Skeleton of the Mouse. XXVI. Pintail. Genetical Research, 1, 439. Charnley, J. (1952): The Imbibition of Fluid as a Cause of Herniation of the Nucleus Pulposus. Lancet, i, 124.

Eckert, C., and Decker, A. (1947): Pathological Studies of Intervertebral Discs. Journal of Bone and Joint Surgery, 29, 447.

GrüneberG, H. (1953): Genetical Studies on the Skeleton of the Mouse. VII. Congenital Hydrocephalus. Journal of Genetics, 51, 327.

Grüneberg, H. (1958): Personal communication.

Hansen, H.-J. (1951): A Pathologic-anatomical Interpretation of Disc Degeneration in Dogs. Acta Orthopaedica Scandinavica, 20, 280.

Hansen, H.-J. (1952): A Pathological-anatomical Study of Disc Degeneration in Dog. Acta Orthopaedica Scandinavica, Supplementum 11.

Hendry, N. G. C. (1958): The Hydration of the Nucleus Pulposus and its Relation to Intervertebral Disc Derangement. Journal of Bone and Joint Surgery, 40-B, 132.

Hollander, W. F., and Strong, L. C. (1951): Pintail, a Dominant Mutation Linked with Brown in the House Mouse. Journal of Heredity, 42, 179.

JaCkson, H. (1948): The Association Between Certain Anatomical Facts, Normal and Morbid, and the Symptomatology of Intervertebral Disc Protrusions in the Lumbar Region. Annals of Royal College of Surgeons of England, 2, 273.

Lancet (1958): Annotation: Physical Changes in the Prolapsed Disc, i, 1,214.

Lison, L. (1954): Alcian Blue 8G with Chlorantine Fast Red 5B. A Technic for Selective Staining of Mucopolysaccharides. Stain Technology, 29, 131.

MaLINSKÝ, J. (1957): Histochemical Demonstration of Carbohydrates in Human Intervertebral Discs During Postnatal Development. Acta Histochemica, 5, 120.

MALINSKÝ, J. (1959): Histochemistry of Carbohydrates and Lipids in Intervertebral Discs During Phylogenetic Development. Acta Histochemica, 7, 107.

Mitchell, P. E. G., Hendry, N. G. C., and Billewicz, W. Z. (1961): The Chemical Background to Intervertebral Disc Prolapse. Journal of Bone and Joint Surgery, 43-B, 141.

Naylor, A. (1958): Changes in the Human Intervertebral Disc with Age. Proceedings of Royal Society of Medicine (Section of Comparative Medicine), 51, 573.

Nоваск, G. J. (1916): The Use of the Van Wijhe Method for the Staining of the Cartilaginous Skeleton. Anatomical Record, 11, 292.

PeAcock, A. (1952): Observations on the Post-natal Structure of the Intervertebral Disc in Man. Journal of Anatomy, 86, 260.

Sylvén, B. (1951): On the Biology of Nucleus Pulposus. Acta Orthopaedica Scandinavica, 20, 275.

VOL. 43 B, NO. 2, MAY 1961 